\title{
$\operatorname{ReGenX}$ \\ BIOSCIENCES
}

\section{ReGenX AAV Vector Technology: a tool for in vivo screening}

Transgenic and knockout mice have been used to create disease models and understand gene function. However, such mice are complex to create, breeding colonies must be established, and some models are not viable. Somatic gene transfer with vectors such as adeno-associated virus (AAV) can overcome these limitations. ReGenX's AAV Vector Technology gives extremely efficient and stable gene expression in neonatal and adult animals, and can be used to create disease models and to study gene function and target validation.

\begin{abstract}
AAV as a somatic gene transfer vector
ReGenX's AAV Vector Technology offers highly effective vehicles for in vivo gene transfer. AAV is a parvovirus, consisting of only capsid protein and a single-stranded DNA genome ${ }^{1}$. AAVs are nonpathogenic and naturally replication defective, requiring helper genes from another virus to replicate. The AAV genome consists of two inverted terminal repeats (ITRs) flanking the two AAV genes: rep, encoding replication proteins, and cap, encoding capsid proteins. Recombinant AAV (rAAV) is created by deleting both rep and cap and inserting an expression cassette between the two ITRs. These recombinant, replicationdefective AAVs are able to enter cells and deliver their DNA genome to the nucleus, but are unable to replicate. Most of the rAAV genomes do not integrate; instead, they are maintained episomally. In nondividing or slowly dividing cells in vivo, transgene expression can persist for years.
\end{abstract}

\section{ReGenX AAV Vector Technology}

The original AAV serotypes, AAV1 through AAV6, although useful, were not efficient at transducing many major tissue targets in vivo. New AAV capsid sequences were isolated from human and nonhuman primate tissues, cloned to generate recombinant AAV vectors and characterized in multiple tissues in vivo ${ }^{2,3}$. The resulting ReGenX AAV Vector Technology, which includes serotypes AAV7, AAV8 and AAV9, stood out by virtue of being 1-2 logs more efficient than AAV2 in delivering genes to multiple rodent tissues. Importantly, these serotypes maintained the stability of gene expression in the absence of inflammation.

In liver, all three of the above serotypes are more efficient than

\section{Karen Kozarsky}

ReGenX Biosciences, Washington, DC, USA

Correspondence should be addressed to K.K. (kkozarsky@regenxbio.com)

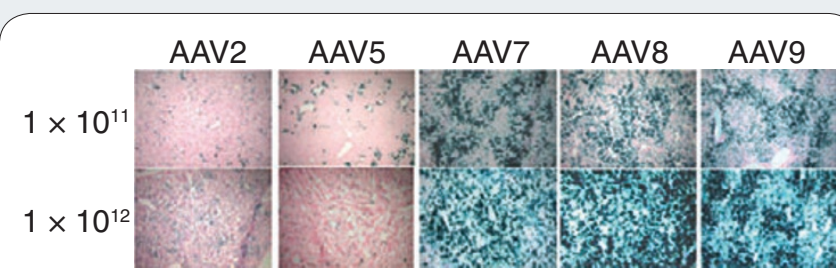

Figure 1 | Gene transfer to liver. Recombinant AAV encoding the $\beta$-galactosidase ( $L a C Z$ ) gene was injected intravenously into mice at the indicated doses. Livers were harvested 90 d post-injection, sectioned, stained with X-gal (blue) and counterstained with hematoxylin. Courtesy of Gene Therapy Program, University of Pennsylvania.

AAV1-AAV6 in rodents (Fig. 1). In muscle, direct injection of AAV8 yields transgene expression levels at least 2 logs higher than injection of AAV2 (ref. 4), and in heart tissue, AAV9 injected into newborn mice results in transduction throughout the heart, which persists for at least 1 year ${ }^{5}$. In the brain, AAV9 and AAVrh. 10 both give high levels of transgene expression as well as a much wider distribution of transduction than other serotypes ${ }^{6}$. Characterization of the novel AAV serotypes as well as the traditional serotypes has led to the selection of optimal AAV serotypes for different tissues (see Fig. 2). It is also

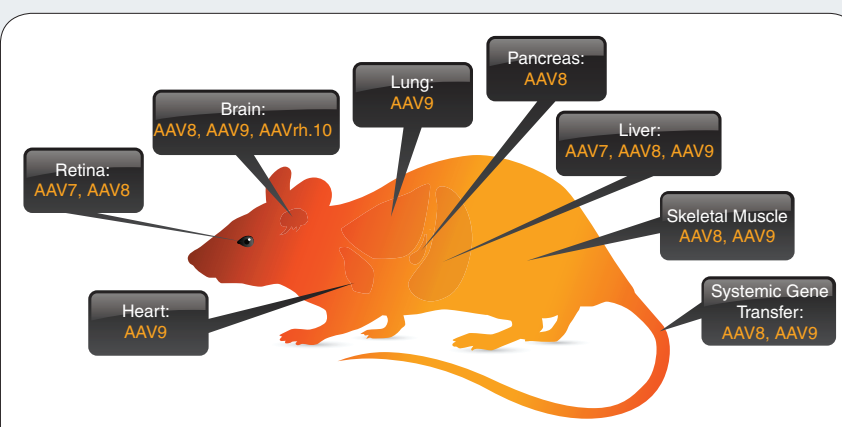

Figure $\mathbf{2}$ | Optimal AAV serotypes for in vivo gene transfer to various tissues. 
possible to target specific tissues through choice of serotype, route of administration and promoter selection.

\section{Generating disease models}

AAV can be used to introduce genes or to knock out gene expression in neonatal or adult mice to induce a disease state. Such mice can be used to more fully understand the pathways of disease, or as a disease model for testing therapeutics, whether small-molecule or biologic. For example, AAV has been used to express a short hairpin RNA (shRNA) in the hypothalamus to generate a mouse model of obesity ${ }^{7}$. In addition, an AAV-induced knockout of vascular endothelial growth factor (VEGF) in the lung generated a model of emphysema ${ }^{8}$. This model was created in a VEGF-loxP transgenic mouse, in which VEGF expression was specifically eliminated in the lung by tracheal installation of an AAV encoding the Cre recombinase. The Cre-loxP approach can be used in most major tissues to generate tissue-specific knockouts.

\section{Screening therapeutic proteins in vivo}

Screening therapeutic proteins in animal models can be difficult and time-consuming. To avoid the different purification methods and repeated in vivo protein injections, it is possible to clone cDNAs into recombinant AAV vectors and inject mice with rAAV. For example, AAV8 can be injected intravenously to achieve high circulating levels of a secreted protein. This technique has been used to determine which variants of apolipoprotein A-I (ApoA-I) are most effective in preventing atherosclerosis ${ }^{9}$. A similar technique has been used to demonstrate efficacy of monoclonal antibodies. AAV8 encoding an antibody to the VEGF receptor was injected into mice, and injection of glioblastoma cells resulted in significant reductions in tumor size and an increased rate of survival in mice expressing the monoclonal antibody ${ }^{10}$.

\section{Conclusion and perspective}

ReGenX's AAV Vector Technology delivers genes in vivo with high efficiency, with a faster onset and higher levels of gene expression than previously characterized AAV serotypes. One can target tissues through a combination of the AAV serotype selected, the route of administration and the use of tissue-specific promoters. This technology can be used to overexpress genes in vivo or to inhibit gene expression. It has broad utility in understanding gene function, as it offers the ability to express the gene of choice at the desired time. New animal models can be created much faster than with traditional transgenic or knockout technologies, and potentially therapeutic proteins can be screened rapidly in vivo. ReGenX provides AAV with reporter genes or custom genes to help achieve each project's goal.

\section{ACKNOWLEDGMENTS}

We thank J.M. Wilson for a critical reading of the manuscript, and the University of Pennsylvania Gene Therapy Program for data included in Figure 1.

1. Muzyczka, N. \& Burns, K.I. Parvoviridae: the viruses and their replication. in Fields Virology 4th edn. (Howley, P.M. \& Knipe, D.M.) 2327-2360 (Lippincott, Williams and Wilkins, Philadephia, 2001).

2. Gao, G. et al. Clades of Adeno-associated viruses are widely disseminated in human tissues. J. Virol. 78, 6381-6388 (2004)

3. Gao, G. et al. New recombinant serotypes of AAV vectors. Curr. Gene Ther. $\mathbf{5}$, 285-297 (2005).

4. Ohshima, S. et al. Transduction efficiency and immune response associated with the administration of AAV8 vector into dog skeletal muscle. Mol. Ther. 17, 73-80 (2009).

5. Bish, L.T.et al. Adeno-associated virus (AAV) serotype 9 provides global cardiac gene transfer superior to AAV1, AAV6, AAV7, and AAV8 in the mouse and rat. Hum. Gene Ther. 19, 1359-1368 (2008).

6. Cearley, C.N. \& Wolfe, J.H. Transduction characteristics of adeno-associated virus vectors expressing cap serotypes 7,8,9, and Rh10 in the mouse brain. Mol. Ther. 13, 528-537 (2006)

7. Garza, J.C. et al. Adeno-associated virus-mediated knockdown of melanocortin-4 receptor in the paraventricular nucleus of the hypothalamus promotes high-fat diet-induced hyperphagia and obesity. J. Endocrinol. 197, 471-482 (2008).

8. Tang, K. et al. Lung-targeted VEGF inactivation leads to an emphysema phenotype in mice. J. Appl. Physiol. 97, 1559-1566 (2004).

9. Lebherz, C. et al. Gene transfer of wild-type apoA-I and apoA-I Milano reduce atherosclerosis to a similar extent. Cardiovasc. Diabetol. 6, 15 (2007).

10. Fang, J. et al. Stable antibody expression at therapeutic levels using the $2 \mathrm{~A}$ peptide. Nat. Biotechnol. 23, 584-590 (2005).

This article was submitted to Nature Methods by a commercial organization and has not been peer reviewed. Nature Methods takes no responsibility for the accuracy or otherwise of the information provided. 